\title{
IMPACTO DEL DESARROLLO DE HÍBRIDOS DE MAÍZ EN CENTRO AMÉRICA ${ }^{1}$
}

\author{
H. S. Córdova, H. J. Barreto y J. Crossa
}

\begin{abstract}
RESUMEN
Impacto del desarrollo de híbridos de maíz en Centro América. En este trabajo se analiza el progreso alcanzado en el desarrollo de híbridos de maíz en Centro América utilizando como fuente la base de datos regional del PCCMCA (Programa Colaborativo Centroamericano para el Mejoramiento de Cultivos y Animales) y tomando como referencia 51 ensayos realizados entre 1988 y 1990. Esta evaluación se 1Ievó a cabo utilizando una metodología que determina la confiabilidad de respuesta $\left(\mathrm{RN}_{\mathrm{i}}\right.$ o probabilidad de respuesta normalizada superior a cero) de los cultivares superiores con respecto a un cultivar testigo. Se determinó la confiabilidad de los cultivares (HB83, HB85, H30, HA46, Dekalb B833, Pioneer XLH53, HRI7, MAX307, H33 y TACSA 203) como una medida que integra tanto la magnitud de respuesta como la variabilidad en rendimiento con respecto al testigo $\mathrm{H} 5$. Los geno tipos evaluados se clasificaron en tres clases con respecto al H5. Genotipos de confiabilidad Superior (H30 y HB85 0,9 >= RNi $<=1,0$ ), confiabilidad Buena (HB83, HA46, DekB833,XLH53, $0,8>=\mathrm{RNi}<0,90$ ) y confiabilidad Promedio (HRI7, MAX307, H33, 0,6 >= RNi < 0,8). Entre los genotipos que mostraron mayor potencial como testigos regionales se encuentran el HB85 y HB83. Se concluye que se necesitan mayores estudios para correlacionar el uso de la confiabilidad con otros métodos para determinar la estabilidad genotípica.
\end{abstract}

\begin{abstract}
Impact ofthe coro (Zea mays L) hybrids' development in Central America. The progress achieved by the corn hybrids' development in Central America is analyzed as the source for the regional data base of the PCCMCA (Programa Colaborativo Centroamericano para el Mejoramiento de Cultivos y Animales), taking as reference the 51 trials conducted between 1988 and 1990. This evaluation was conducted using a methodology which determines the confidence of response $\left(\mathrm{RN}_{\mathrm{i}}\right.$ or probability of normalized response above zero) of superior cultivars with respect to a control. The confidence of the cultivars (HB83,HB85, H30, HA46, Dekalb B833, Pio-neer XLH53, HRI7, MAX307, H33 and TACSA203) was determined as a measure which integrates the magnitude of response as we1l as the variability of yields, with respect to the control H5. The evaluated genotypes were divided into three cIasses with respect to the H5. Genotypes of Superior confidence (H30 and HB85, 0.9>= $\mathrm{RN}_{\mathrm{i}}<=1.0$ ), Good confidence (HB83, HA46, Dekalb B833, XLH53, $0.8>=\mathrm{RN}_{\mathrm{i}}<0.90$ ) and Average confidence (HRI7, MAX307, H33, $0.6>=\mathrm{RN}_{\mathrm{i}}<$ 0.8). The genotypes HB85 and HB83 showed the highest potential as regional controls. In conclusion, further studies are needed to correlate the use of confidence to other methods in order to determine the genotype stability.
\end{abstract}

\section{INTRODUCCION}

A partir de la década de los 1980, la evaluación experimental del maíz híbrido en relación a las variedades de polinización libre (VPL) ha recibido mayor atención por los fitomejoradores del área Centroamericana en la búsqueda continua de alternativas para aumentar la producción de maíz, particularmente en ambientes favorables. La generación de híbridos con alto potencial de rendimiento y amplia adaptabilidad a los diferentes ambientes de producción requiere en primera instancia de un programa de mejoramiento con objetivos precisos y de una eficiente infraestructura para la producción de semilla. Asimismo, se reconoce que para explotar el potencial genético de los híbridos, los aspectos agronómicos de manejo del cultivo (e.g. densidad, fertilización, etc) revisten una importancia

\footnotetext{
1 Presentado en la XXXIX Reunión Anual del PCCMCA en Guatemala, América Central. 28 de marzo - 3 de abril, 1993.

2 Respectivamente, Fitogenetista, Agrónomo y Biometrista. Centro Internacional de Mejoramiento de Maíz y Trigo, (CIMMYT). Apdo. Postal 6-641 CP 06600 México D. F. México.
} 
aún mayor que en las VPL. El desarrollo de híbridos estables y de alta producción ha facilitado la incorporación de otros componentes (fertilizantes y herbicidas) a los sistemas de producción, contribuyendo así al aumento de la productividad del cultivo del maíz de la región.

Durante el período 1966-1980, las VPL representaron mas del $70 \%$ del germoplasma producido por los programas nacionales de investigación de Maíz de Centro América y el Caribe (Morris y Lopez-Pereira, 1991; Lopez-Pereira, 1993). Para el período 1980-1990, se observa una tendencia mayor al desarrollo de híbridos (Córdova, 1991; Lopez-Pereira, 1993). Entre los factores importantes que afectan el énfasis actual de la investigación en fitomejoramiento de maíz en la región de México y Centroamérica, Lopez-Pereira y Espinosa (1993) mencionan la productividad del híbrido, rendimiento de las líneas progenitoras, el grado de eficiencia en las diferentes etapas de proceso de producción de semilla, y el impacto de las políticas agrícolas. Córdova (1989) resalta la necesidad de una mayor integración de los programas de mejoramiento genético a los sistemas de generación, prueba y transferencia de tecnología para una eficiente diseminación de los genotipos generados en la región. Lopez-Pereira (1993) indicó una relación inversa entre el precio relativo y el uso de semilla híbrida por los agricultores en países en vías de desarrollo.

El ensayo uniforme de cultivares de maíz del Programa Cooperativo Centroamericano para el Mejoramiento de Cultivos y Animales (PCCMCA) se inició en 1956, como un instrumento colaborativo para la evaluación sistemática de cultivares desarrollados por los Programas Nacionales y las empresas privadas que operan en la región. La experiencia acumulada ha permitido fortalecer las técnicas de campo para la realización de experimentos así como el análisis e interpretación de los resultados mediante el uso de metodologías apropiadas para lograr conclusiones acertadas. La base de datos histórica de los ensayos colaborativos ha permitido a los programas nacionales tomar decisiones y reorientar los sistemas de mejoramiento del maíz en la región. La mayoría de los cultivares de maíz en producción comercial en la región han tenido como base de su liberación el ensayo del PCCMCA, desde los híbridos H3 y H5 liberados en El Salvador en 1963-1965 hasta los híbridos HB83M y HB85 liberados en Guatemala en 1989. En síntesis, el ensayo colaborativo PCCMCA ha probado ser un instrumento efectivo para la evaluación experimental de los mejores materiales disponibles en el mercado de semilla de la región.

El Programa Regional de CIMMYT para Centro América y el Caribe estableció un proyecto colaborativo regional encaminado a la generación de híbridos en 1986. En 1989 se inició la evaluación experimental a nivel regional a través del ensayo colaborativo del PCCMCA. Los primeros híbridos resultantes de este esfuerzo culminaron con la liberación de nuevos materiales en Panamá y Costa Rica en 1990 y 1991, respectivamente. El progreso fitogenético y la amplia adaptabilidad a ambientes de producción de los híbrido s generados en Centro América durante 1986-1990 han sido documentados por Córdova (1991)

Específicamente, Córdova (1991) en una revisión comprensiva de los resultados experimentales de los ensayos del PCCMCA 1988-1990, indica que los híbridos HB85 y HB83 superaron al testigo histórico H5 en forma concluyente. Además indica que existen híbridos de maíz generados por las compañías privadas que pueden competir con los materiales desarrollados por los programas nacionales de investigación. Las conclusiones de estos análisis con respecto a la interacción genotipoambiente permiten: 1. asumir una adaptabilidad amplia (regional) de algunos de los genotipos y 2. una evaluación del progreso genético alcanzado en los últimos años. Sin embargo, se hace necesario realizar un inventario apropiado de la magnitud y variabilidad de respuesta observada en genotipos selectos con el fin de incorporarlos en forma eficiente a los paquetes tecnológicos disponibles en la región. 
Los objetivos de este trabajo son:

1. Cuantificar la probabilidad de ganancias específicas en rendimiento de diez híbridos de maíz en comparación al testigo histórico $\mathrm{H} 5$.

2. Plantear algunas consideraciones para la selección de testigos a ser utilizados en comparaciones a nivel regional en estudios de impacto y ganancia genética.

3. Introducir la metodología de confiabilidad de respuesta contra un testigo para caracterizar genotipos.

\section{MATERIALES Y MÉTODOS}

\section{Fuente de Datos Experimentales}

Las localidades y la técnica experimental de los ensayos colaborativos PCCMCA utilizados en este trabajo han sido descritas por Córdova (1991). Para este estudio se utilizaron ensayos efectuados en 51 localidades como parte del proyecto colaborativo de evaluación de híbridos del PRM a través del PCCMCA, de estos se seleccionó un juego de genotipos comunes evaluados durante los años 1988-1990. Sin embargo, algunos genotipos no fueron evaluados en la totalidad de sitios. Los genotipos considerados, su origen y el número de localidades consideradas se presentan en el Cuadro 1. Los genotipos seleccionados representan un grupo de materiales comúnmente utilizados en la región y comprenden tanto materiales generados por los programas de mejoramiento de los países del área en colaboración con el CIMMYT, al igual que algunos materiales representativos del germoplasma generado por la industria semillerista privada.

\section{Conflabilidad de Respuesta}

Para determinar la confiabilidad de respuesta de un genotipo cualquiera (i) con respecto al testigo histórico H5 se utilizó una modificación del método de Eskridge y Mumm (1992). La confiabilidad de respuesta del iecimo genotipo se determinó a partir de los diferenciales de rendimiento con respecto al testigo $\mathrm{H} 5\left(\mathrm{~d}_{\mathrm{i}}\right)$. La hipótesis a probar en este caso es si el valor promedio de di es mayor que cero, o sea:

$$
\begin{gathered}
\mathrm{H}_{\mathrm{o}}: \mathrm{d}<=0 \\
- \\
\mathrm{H}_{\mathrm{a}}: \mathrm{d}_{\mathrm{i}}>0
\end{gathered}
$$

Para probar esta hipótesis se calculan los valores pro-

Cuadro 1. Nombre del híbrido, origen, color de grano y número de localidades de evaluación en Centroamérica, 1988-1990

\begin{tabular}{lllll}
\hline $\begin{array}{l}\text { Nombre del } \\
\text { híbrido }\end{array}$ & $\begin{array}{l}\text { Institución } \\
\text { /compañía }\end{array}$ & Pais de origen & $\begin{array}{c}\text { Color de } \\
\text { grano }\end{array}$ & $\begin{array}{c}\text { número de } \\
\text { ambientes }\end{array}$ \\
\hline Dekalb B-833 & Dekalb & U.S.A. & Blanco & 31 \\
H-30 & DIA & Honduras & Blanco & 51 \\
H-33 & DIA & Honduras & Blanco & 34 \\
H-5 & CENTA & El Salvador & Blanco & 51 \\
HA-46 & ICTA & Guatemala & Amarillo & 51 \\
HB-83 & ICTA & Guatemala & Blanco & 51 \\
HB-85 & ICTA & Guatemala & Blanco & 51 \\
HR-17 & Seminal & Guatemala & Blanco & 51 \\
MAX-307 & Agridec & U.S.A. & Blanco & 51 \\
TACSA 203 & TACSA & Mexico & Amarillo & 51 \\
XLH53 & Pioneer & U.S.A & Amarillo & 51 \\
\hline
\end{tabular}


medio de $\mathrm{d}_{\mathrm{i}}$ y la desviación estándar de las diferencias a través de todos los sitios $\left(\mathrm{S}_{\mathrm{di}}\right)$. Luego se determina el valor estandarizado $\left(\mathrm{d}_{\mathrm{i}} / \mathrm{s}_{\mathrm{di}}\right)$ al cual se le determina la probabilidad normal estandarizada (usando la tabla de z normal de una cola). A esta probabilidad se le denomina Confiabilidad Normalizada con Respecto al Testigo $\left(\mathrm{RN}_{\mathrm{i}}\right)$. La confiabilidad se calculó para cada comparación de interés y se procedió a ordenar los genotipos en términos de esta probabilidad (de mayor a menor).

Los valores de $\mathrm{RN}_{\mathrm{i}}$ para cada genotipo representan la probabilidad de que un genotipo supere cierto nivel de producción establecido por el híbrido adaptado (H5). Si, para un genotipo $\mathrm{i}$, esta probabilidad es baja $\left(0,5>\mathrm{RN}_{\mathrm{i}}<=\right.$ $0,6)$ quiere decir que, en promedio, dicho genotipo no supera al testigo adaptado, por 10 que su adopción como tecnología alternativa no es recomendable. Por el contrario, si el genotipo i tiene un valor de confiabilidad alto (e.g. $\mathrm{RN}_{\mathrm{i}}>0,8$ ) quiere decir que se espera, en 8 de cada 10 casos en promedio, una respuesta superior al testigo adaptado y por lo tanto su adopción por los agricultores puede ser recomendable.

Cabe destacar que la medida de confiabilidad aquí presentada no cuantifica la magnitud de la superioridad (en términos de ganancias en rendimiento) del nuevo genotipo con respecto al testigo. Es decir, $\mathrm{RN}_{\mathrm{i}}$ no incorpora ningún factor económico asociado al cambio tecnológico. Esto es una consideración importante, ya que los incrementos en rendimientos representados por la adopción de una nueva tecnología (en este caso un nuevo genotipo) no siempre compensan económicamente dicha adopción. Por ejemplo, una ganancia promedio en rendimiento de 250 $\mathrm{kg} /$ ha del genotipo i con una desviación estándar de 500 $\mathrm{kg} / \mathrm{ha}$ de la muestra de diferencias sobre el testigo, se asocia a una confiabilidad de 0,69 , la cual no necesariamente implica la necesidad de un cambio tecnológico.

\section{Confiabilidad Económica de la Respuesta}

La incorporación del factor económico asociado al cambio tecnológico propuesto (de H5 al nuevo híbrido) se puede realizar considerando que la diferencia promedio di supere un determinado nivel de costos. Este nivel estaría relacionado al incremento en rendimiento necesario para cubrir y/o superar los costos del cambio tecnológico (los costos c, están expresados en las unidades de medida del rendimiento). De esta manera la adopción de un nuevo genotipo sería recomendable solo si $\mathrm{d}_{\mathrm{i}}>\mathrm{c}$. Las hipótesis de interés son:

$$
\begin{aligned}
& \overline{\mathrm{H}}_{\mathrm{o}}: \mathrm{d}_{\mathrm{i}}-\mathrm{c}<=0 \\
& -\mathrm{H}_{\mathrm{a}}: \mathrm{d}_{\mathrm{i}}-\mathrm{c}>0
\end{aligned}
$$

Esta probabilidad se obtiene de la variable normal estandarizada $\mathrm{z}$ de una cola y se le denomina $\mathrm{ERN}_{\mathrm{i}}$. De esta forma los genotipos se ordenan de acuerdo a sus valores de $E R N_{i}$ para cada genotipo y costos asociados con un cambio tecnológico en particular.

\section{RESULTADOS Y DISCUSIÓN}

\section{Confiabilidad contra el testigo H5}

El método propuesto está basado en la premisa de que el proceso de decisión sobre selección de un genotipo, sea por los fitomejoradores o por los productores, esta fuertemente influenciado por el nivel de certeza estadística que se tenga, sobre el potencial de los nuevos cultivares de tener una alta probabilidad de superar a un cultivar testigo en una localidad determinada (Nuland y Eskridge, 1991; Eskridge y Mumm, 1992) y de cubrir o superar los costos del cambio tecnológico. En este sentido, se define a un genotipo como de confiabilidad económica superior, como aquel que tiene una alta probabilidad de sobrepasar en comportamiento al testigo dentro del rango de costos de producción asociados al cambio tecnológico. El cultivar con la confiabilidad más alta, dentro de un rango de costos dado, es el mejor cultivar. Entre más pequeña y cercana a 0,5 sea la confiabilidad económica, existe un mayor riesgo asociado al comportamiento del genotipo de prueba con respecto al testigo. 
El híbrido H5 ha sido tradicionalmente utilizado como un comparador para medir el progreso genético de los nuevos cultivares de maíz híbrido en la región de Centro América y El Caribe (Córdova, 1991). El H5 fue inicialmente desarrollado e introducido a los agricultores alrededor de 1963-1965 (Walker, 1980), por lo que establece un punto de referencia histórica. A pesar de algunas limitaciones con respecto a la susceptibilidad de este material con respecto a algunos estreses, particularmente bióticos (e.g. achaparramiento), su potencial de rendimiento y difusión entre los agricultores lo han convertido en un verdadero "caballo de batalla" en la lucha incesante para aumentar la producción de maíz en la región Centroamericana.
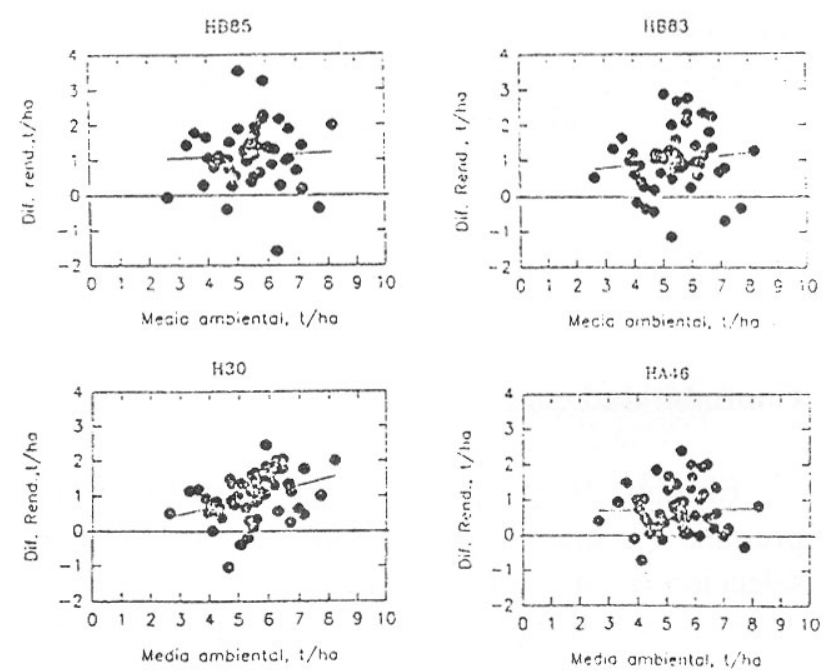

Figura 1. Diagramas de dispersión de las deferencias de rendimiento y la estabilidad relativa de cuatro híbridos de maíz con respecto al testigo histórico H5 evaluados en Centroamérica durante 1988-1990.

Los diagramas de dispersión y regresión lineal de las diferencias de rendimiento con respecto al $\mathrm{H} 5$ de los diez híbridos considerados en este estudio en relación a la media ambiental se presentan en las figuras 1-3. Las confiabiliJades calculadas para los diferentes genotipos evaluados se presentan en el Cuadro 2. Los genotipos (H30 y
HB85) presentaron confiabilidades por encima de 0,90 , las cuales se consideran superiores. La probabilidad normalizada de estos dos genotipos sugiere una repuesta diferencial en rendimienlo mayor quc cero con respecto al H5 en 9 de cada 10 instancias.

Sin embargo, para el agricultor el cambio tecnológico de pasar de un genotipo a otro involucra costos, sean estos directos (semilla más costosa) o indirectos (costos de infraestructura para la producción de nueva semilla y/o subsidios a la semilla). Mediante una modificación al método propuesto por Eskridge y Mumm (1992), nosotros evaluamos el cambio en la probabilidad de respuesta a medida que los costos incurridos en el cambio tecnológico (H5 a híbrido nuevo) aumentan. El Cuadro 2, ilustra este efeclo para los genotipos evaluados bajo diferentes escenarios de costos (en unidades de rendimiento, 0,25, 0,50 y $0,75 \mathrm{t} / \mathrm{ha}$ ) asociados con el calubio tecnológico. Los genotipos se agruparon en tres grupos de acuerdo a su confiabilidad (RN). Confiabilidad Superior (1130 y HB85 0,9 >= RNi <= 1,0), confiabilidad Buena (HUS3, HA46, DekB83,XLH53, 0,8 >= RNi < 0,90) y confiabilidad Promedio (HR17, MAX307, H33, $0.6>=\mathrm{RNi}<$ $0,8)$. El genotipo TACSA-203 presenta una confiabilidad por debajo de 0,6 y por lo tanto representaría un ricsgo relativamente similar para el agricultor con respecto a la utilización del H5.

De la información en Cuadro 2 para los genotipos de confiabilidad superior se observa claramente que sí los costos de cambio tecnológico son cero (lo cual es dudoso) el híbrido H30 presentaría una pequeña ventaja sobre el HB85. Sin embargo, a medida que los costos aumentan por encima de 0,5 t/ha (expresados como toneladas de maíz/ha), el HB85 se hace relativamente más conl'iablc. Esto se explica en función del mayor diferencial de rendimiento del HB85 sobre el H5 a pesar de una mayor varianza con respecto al H30. En forma similar para los híbridos de confiabiliJad Buena, el HB83 representa la alternativa más adecuada pues la probabilidad de respuesta, mín a costos de hasta 0,5 t/ha, permanece siempre por encima de la de los otros materiales. Para los híbridos de con fiabilidad Promedio, sí los costos del cambio tecno- 

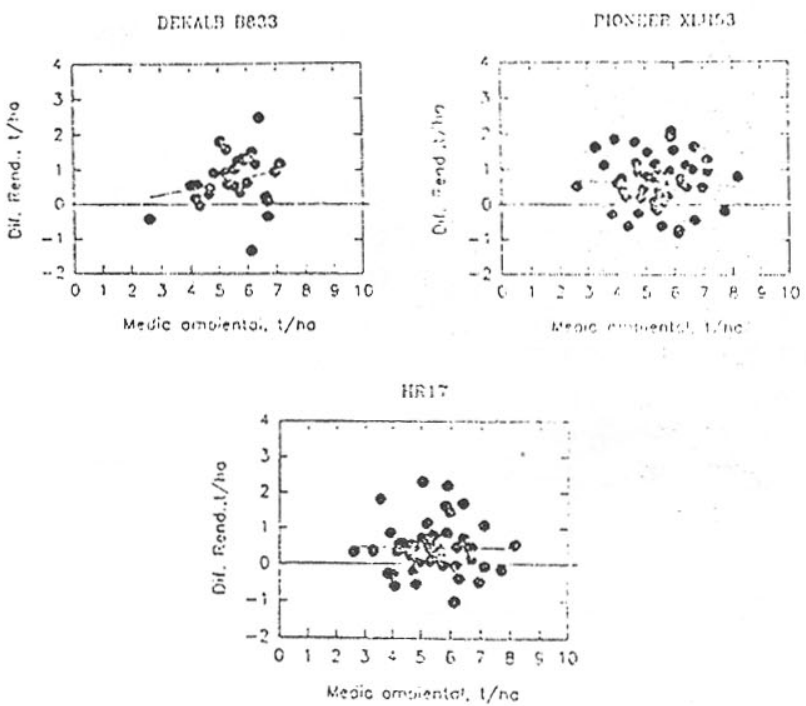

Figura 2. Diagramas de dispersión de las diferencias de rendimiento y la estabilidad relativa de tres híbridos de maíz con respecto al testigo histórico 115 evaluados en Centroamérica durante 1988-1990.
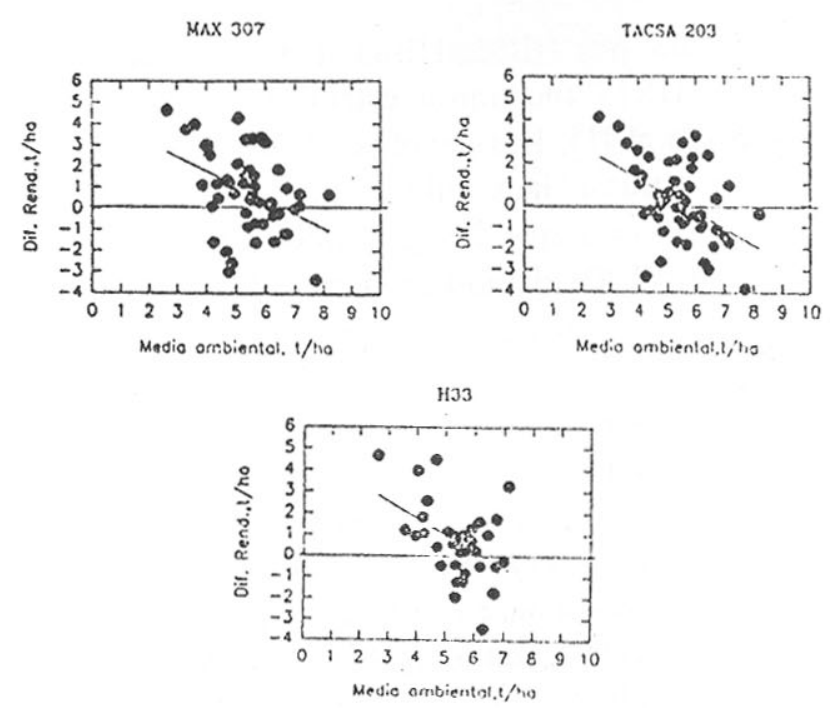

Figura 3. Diagramas de dispersión de las difere ecias de rendimiento y la estabilidad relativa de tres híbridos de maíz con respecto al testigo historico H5 evaluados en Centroamérica durante 1988-1990. lógico son bajos (e.g. $<0,2$ t/ha), la opción obvia sería el HR 17 (RNi =0,76); sin embargo, si los costos se aumentan por encima de este nivel, la con fiabilidad de respuesta para todos los híbridos de este grupo se aproxima a 0,5 y por lo tanto el cambio tecnológico tiene un mayor riesgo en comparación al 115 (e.g. una probabilidad de 0,5 indica que se tiene tan solo una probabilidad de 1:1 o 50\% de que el híbrido rinda por encima del H5).

\section{Análisis de estahilidad relativa}

Con respeclo a la estabilidad de los genotipos evaluados a través de ambientes, Eskridge y Mumm (1992) demostraron que la confiabilidad es una medida que está relacionada a otros parámetros de estabilidad genotípica. Entre los parámetros de estabilidad que han demostrado estar en estrecha correlación se incluyen el coeficiente de regresión de Finlay y Wilkinson (1963), la desviación del cuadrado medio de Eberhart y Rusell (1966) y la varianza de estabilidad de Shukla (1972). En este sentido, la confiabilidad es una medida quc evalua tanto las ganancias en rendimiento con respecto al testigo así como la variabilidad de respuesta (Figura 4). La caracterización de la estabilidad de los genotipos evaluados en este estudio utilizando la metodología de Eberhart y Russel (1966) y el modelo multiplicativo AMMI (Gauch, 1988), han sido documentados por Córdova (1991). Sin embargo, las correlaciones de estos resultados con el método de evaluación propuesto no se incluyen en el presente trabajo y constituyen una área futura de validación del método.

Una manera alternativa de obtener una medida de la estabilidad de los materiales evaluados en este trabajo con respecto al testigo histórico es utilizando la modificación del análisis de estabilidad de Finlay y Wilkinlson (1963) propuesta por Raun, Barreto y Westerman (1993). Mediante esta es posible caracterizar la estabilidad relativa de los diferenciales de rendimiento $\left(\mathrm{d}_{\mathrm{i}}\right)$ con respecto a la media ambiental utilizando regresión lineal. Los resultados del análisis de regresión se presentan en el Cuadro 3. En este último método, la estabilidad de los genotipos con respecto al testigo se caracteriza en función de la pendiente lineal, su signo y la probabilidad de que sea 
Cuadro 2. Promedios de rendimiento, diferencias en rendimiento, desviaciones estándar y confiabilidad económica a diferentes niveles para 10 hibridos de maíz con respecto al H5 en evaluaciones realizadas en 51 ambientes de Centroamérica durante 1988-1990).

\begin{tabular}{|c|c|c|c|c|c|c|c|}
\hline Entrada & $\begin{array}{l}\text { Rend. } \\
\text { t/ha }\end{array}$ & $\begin{array}{l}\text { Iiff. } \\
\text { t/ha }\end{array}$ & $\begin{array}{c}\text { Desv Est. } \\
\text { t/ha }\end{array}$ & $\mathrm{RN}_{i}>0$ & $\underset{c>0,25 t}{\mathrm{ERN}_{i}}$ & $\begin{array}{l}\mathrm{ERN}_{i} \\
\mathrm{c}>0,5 \mathrm{t}\end{array}$ & $\begin{array}{c}\operatorname{ERN}_{i} \\
\mathrm{c}>0,75 t\end{array}$ \\
\hline HB85 & 5,92 & 1,126 & 0,857 & 0,905 & 0,846 & 0,767 & 0,660 \\
\hline HB83 & 5,79 & 0,998 & 0,856 & 0,878 & 0,808 & 0,719 & 0,613 \\
\hline $\mathrm{H} 30$ & 5,77 & 0,977 & 0,674 & 0,926 & 0,859 & 0,760 & $0,6,32$ \\
\hline MAX307 & 5,59 & 0,798 & 1,921 & 0,661 & 0,612 & 0,561 & 0,510 \\
\hline DekB83 & 5,56 & 0,700 & 0,726 & 0,832 & 0,732 & 0,608 & 0,472 \\
\hline HA46 & 5,51 & 0,722 & 0,674 & 0,857 & 0,757 & 0,628 & 0,483 \\
\hline XLH53 & 5,41 & 0,615 & 0,709 & 0,806 & 0,696 & 0,564 & 0,424 \\
\hline H33 & 5,37 & 0,675 & 1,759 & 0,619 & 0,595 & 0,539 & 0,482 \\
\hline HR 17 & 5,26 & 0,467 & 0,672 & 0,756 & 0,627 & 0,480 & 0,337 \\
\hline TACS $\triangle 203$ & 4,94 & 0,149 & 1,854 & $(0,532$ & 0,478 & 0,425 & 0,373 \\
\hline H5 & 4,79 & 0,000 & - & - & - & - & - \\
\hline
\end{tabular}
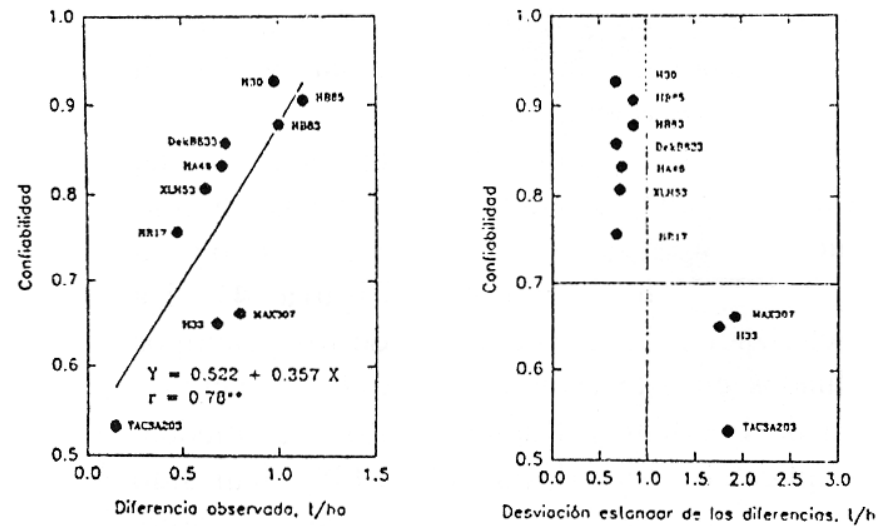

Figura 4. Relación de la confiabilidad con la diferencia en rendimiento contra $\mathrm{H} 5$ y la desviación estándar de la muestra para diez híbricios de maíz evaluados en 51 ambicntes de Centroamérica durante 1988-1990.

diferente de cero. En este contexto, un genotipo estable con respecto al testigo, se define como aquel en el que la pendiente no es significativamentc difercnte de cero. Un genotipo caracterizado como inestable con respecto al testigo (aquel con pendientes fuertemente positivas o negativas), no implica que deba ser excluido o que sea inferior, sino simplemente indica que los diferenciales de rendimiento en relación al testigo se comportan con un grado relativamente alto de interacción con el ambiente.

Los genotipos HB85, HB83, HA46, Pioneer XLH53 y HR17 mostraron estabilidad relativa con respecto al H5. Los genotipos H30 y Dekalb B833 demostraron inestabilidad relativa de tipo positivo, mientras que los genotipos MAX307, H33 y TACSA203 demostraron inestabilidad relativa de tipo negativo con respecto al HS en ambientcs favorables, pero compensaron en alguna manera por su comportamiento sobresaliente en ambientes de menor potencial de rendimiento $(<4 \mathrm{t} / \mathrm{ha})$. Las pendientes de regresión para los genotipos clasificados como estables e inestables se presentan en el Cuadro 3. Los genotipos clasificados como estables (en términos relativos) con la presente metodología también lo fueron en términos absolutos en los estudios de Córdova (1991) y Queme y Fuentes (1992) utilizando la metodología de Eberhart y Russel (1966) y el modelo multiplicativo AMMI (Gauch, 1988). 
Cuadro 3. Pendientes de regresión lineal y prueba de t (pendiente diferente de cero), para las diferencias de rendimiento con respecto al H5 contra la media ambiental, en 51 ambientes de Centroamérica. 1988-1990.

\begin{tabular}{llll}
\hline Híbrido & $\begin{array}{c}\text { Pendiente } \\
\text { de regresión }\end{array}$ & $\begin{array}{c}\text { t estadistico } \\
\mathbf{B}<>\mathbf{0}\end{array}$ & $\begin{array}{l}\text { Prob. } \\
\mathbf{t}<>\mathbf{0}\end{array}$ \\
\hline HB85 & 0,0306 & 0,282 & 0,78 \\
H30 & 2,638 & 0,01 \\
HB83 & 0,2107 & 0,775 & 0,44 \\
HA460,0133 & 0,0836 & 0,88 & 0,20 \\
DekB83 & 0,156 & 1,304 & 0,75 \\
XLH53 & 0,1733 & 0,326 & $\left.0,004^{(}\right)$ \\
MAX307 & $-0,0293$ & 3,034 & 0,007 \\
H33 & $-0,6772$ & 2,906 & 0,95 \\
HR17 & $-0,7826$ & 0,063 & $0,001 @$ \\
TACSA203 & $-0,00532$ & 3,707 & \\
\hline
\end{tabular}

@: indica híbridos con alto grado de interacción con el ambiente respecto al testigo H5.

\section{Consideraciones para la selección de testigos regionales}

La identificación de un testigo en un programa de mejoramiento de carácter regional, cuyo enfoque está orientado al desarrollo de genotipos adaptados a ambientes contrastantes que incluyen factores adversos (bióticos y abióticos) a la producción, es un paso esencial para poder determinar las ganancias genéticas relativas a través del tiempo. Córdova (1991) indica que en un programa de mejoramiento avanzado se debe mantener también un testigo histórico que para la región de Centroamérica lo constituye el H5. Por lo tanto se recomienda su continua inclusión en los ensayos colaborativos PCCMCA.

En su presentación del método, Eskridge y Mumm (1992) advierten claramente y con justa razón sobre los riesgos de utilizar un genotipo testigo que no se encuentre bien adaptado a los ambientes de prueba, debido a que podría proveer estimados de confiabilidad no realistas. En nuestro caso la selección del H5 como testigo está justificada en virtud de la amplia difusión de este material entre los agricultores de la región de Centro América y El Caribe. Por otra parte, para poder medir el progreso genético de los nuevos materiales se hace necesario seleccionar un testigo con características sobresalientes y poca inte- racción con el ambiente. Los resultados de este trabajo sugieren a los cultivares HB85 y HB83 como genotipos que reúnen características adecuadas para ser testigos regionales en evaluaciones futuras de ganancias en rendimiento e impacto a nivel Centroamericano.

Algunos otros problemas adicionales que afectan la selección de un testigo regional son: 1 . el potencial de los nuevos genotipos de sobrepasar en rendimiento en forma consistente al genotipo testigo a través de ambientes y años y 2. la capacidad de la industria semillerista de proveer semilla en cantidades suficientes de los nuevos materiales. En primera instancia, para poder declarar que un genotipo es superior a otro como testigo se hace necesario demostrar no solo que rinde substancial mente más, pero también se hace necesario calificar su estabilidad (variación) en comportamiento a través de ambientes. La Figura 4 presenta la relación observada entre magnitud de la diferencia con respecto al $\mathrm{H} 5$ y la confiabilidad de respuesta de los genotipos evaluados. Es claro, que a mayor diferencial de rendimiento existe una mayor confiabilidad y que en general alrededor de $0,8-1,0$ t/ha la confiabilidad es alta $(>0,8)$. La variación en confiabilidad para los genotipos dentro de un diferencial de rendimiento dado, se debe fundamentalmente a la diferente magnitud de las desviaciones estándar a través de localidades. 
Un testigo regional debe proveer tanto al mejorador como al productor un termino de referencia común para comparar nuevos genotipos. Por lo tanto se requiere que su comportamiento ante todo sea estable y que tenga un nivel adecuado de producción. Estas dos características se pueden obtener de los análisis presentados, ya que los genotipos estables presentan pendientes de regresión de las diferencias de rendimiento (con respecto al testigo) que no difieren significativamente de cero y la confiabilidad caracteriza la probabilidad normal de que la respuesta en rendimiento sea superior al testigo.

\section{CONCLUSIONES}

En este trabajo se determinó la confiabilidad de los cultivares (HB83, HB85, H30, HA46, DekB833, XLH53, HR17, MAX307 y H33) como una medida que integra tanto la magnitud de respuesta y la variabilidad en rendimiento con respecto al testigo H5. Los genotipos evaluados se clasificaron en tres clases con respecto al H5. Genotipos de confiabilidad Superior (H30 y HB85 0,9 >= RNi $<=1,0$ ), confiabilidad Buena (HB83, HA46, DekB833 y XLH53, 0,8 >= RNi <0.90) y confiabilidad Promedio (HR17, MAX307 y H33, 0,6 >= RNi <0,8).

Se identificaron dos genotipos (HB85 y HB83) que poseen características deseables que los califican para su inclusión como testigos regionales en el ensayo del PCCMCA. 1. estabilidad con respecto al testigo histórico H5 y 2. alto potencial de rendimiento en diferentes ambientes de evaluación.

El genotipo $\mathrm{H} 5$ se debe continuar usando como testigo histórico en los ensayos del PCCMCA dada la amplia difusión de este material entre los agricultores de Centro América y El Caribe.

Se necesitan mayores estudios para cor relacionar el uso de la confiabilidad con otros métodos para determinar la estabilidad genotípica.

\section{LITERATURA CITADA}

CÓRDOVA, H. S. 1989. Evaluación de 36 cultivares de maíz en 20 ambientes de Centroamérica, Panamá y El Caribe, PCCMCA 1988. Presentado en la XXXV Reunión anual del PCCMCA, San Pedro Sula, Honduras. Abril 2-7, 1989.

CORDOVA, H. S. 1991. Respuestas diferenciales para rendimiento de híbridos de maíz evaluados en ambientes constrastantes de latinoamerica, PCCMCA 1990. En: Desarrollo y Mejoramiento de Germoplasma para Resistencia a Factores Adversos Bióticos y Abióticos 1990. Publicación del Programa Regional de Maíz para Centro américa y El Caribe. mimeo. pp15-33.

EBERHART, S. A.; RUSSELL, W. A. 1966. Stability parameters for comparing varieties. Crop Sci. 6:36-40.

ESKRIDGE K. M.; MUMM, R.F. 1992. Choosing cultivars based on the probability of outperforming a check. Theoretical and Applied Genetics. 84:494-500.

FINLAY, K. W.; WILKINSON, G. N. 1963. The analysis of adaptation in a plant breeding programme. Aust. J. Agric. Res. 14:742-754.

QUEME, J. L.; FUENTES, M. R. 1992. Evaluación de híbridos de maíz (Zea mays L.) de grano blanco y amarillo en diferentes ambientes de Mexico, Centro America, EL Caribe y Venezuela. Programa Regional de Maiz PRM. Sintesis de resultados experimentales 1991. Vol 3:11-30.

GAUCH, H. G. 1988. Model selection and validation for yield trials with interaction. Biometrics 44(3):705-715.

LOPEZ-PEREIRA, M. A. 1991. Efectos de cambios de políticas agrícolas en la investigación en mejoramiento de maíz en Centro América y México. Trabajo presentado en la XXXIX Reunión Anual del PCCMCA, Marzo 28-AbriI3, 1993. Guatemala.

LOPEZ-PEREIRA, M. A.; ESPINOZA, A. 1993. Análisis económico de la producción y uso de semilla mejorada de maíz: El caso de Mexico. In: Memoria Anual en la XXXIX Reunión Anual del PCCMCA, Marzo 28-Abril3, 1993. Guatemala. 
MORRIS, M. L.; LOPEZ-PEREIRA, M. A. 1991. Impacts of CIMMYT's maize breeding research: A preliminary overview. Borrador. Mexico D.F.: CIMMYT.

NULAND, D. S.; ESKRIDGE, K. M. 1992. Probability of outperforming a check. Annual report of the Bean Improvement Cooperative. Vol 35:17-18.

RAUN W. R.; BARRETO, H. J.; WESTERMAN, R. L. 1993. Use of stability analysis for long term soil fertility experiments. Agron. J. 85:159-167.
SHUKLA, G. K. 1972. Some statistical aspects of partition-ing genotype-environment components of variance. Heredity 29:237-245.

WALKER. 1980. Decision making by Farmers and by the National Agricultural Research Program on the Adoption and Development of Maize Varieties in El Salvador. Ph.D Dissertation, Food Research Institute, Stanford University. 